\title{
Medical malpractice in Iran: A systematic review
}

\author{
Pouran Raeissi ${ }^{1}$, Masood Taheri mirghaed*1, Razieh Sepehrian ${ }^{1}$, Mahnaz Afshari ${ }^{1}$, \\ Mohammad Reza Rajabi
}

Received: 9 Sep 2018

\author{
Published: 14 Oct 2019
}

\section{Abstract}

Background: Medical malpractice represents a serious problem in the health system and is one of the risk factors for patient safety which damages the patient and increases costs for the patient and the health care provider. The importance of these complaints against physicians is that litigation may continue for a long time and become problematic for patients and physicians. The objective of this study was to investigate the complaints of medical malpractices which were referred to Iran Medical Council to provide solutions to reduce the complaints and improve service delivery.

Methods: Embase, PubMed/MEDLINE, ISI/Web of Science (WOS), Scopus, and Iranian databases, such as MagIran, SID, and Irandoc, were searched from 01/01/1990 to 07/01/2018. Also, the grey literature (via Google Scholar) was searched. Studies written in English or in Persian were searched, and keywords used included malpractice, negligence, medical malpractice, physician impairment, Iran, and professional impairment.

Results: Nearly 1455 complaints (36\%) of the total number of 3977 complaints were proved to be medical malpractice, and physicians were acquitted in $2542(64 \%)$ cases. Most complaints were from gynaecologists, accounting for $43 \%$ of all complaints, followed by orthopaedic specialists who ranked second with $21.4 \%$ of the total complaints. The most type of failure was due to lack of skill (30.4\%), followed by negligence (29.2\%). Imprudence or indiscretion (26.3\%) and noncompliance with governmental requirements (14.1\%) were also in the next ranks, respectively.

Conclusion: Improving patient and physician relationships, observing medical ethics, increasing the scientific and technical skills of the medical staff, and following the guidelines and medical protocols will prevent medical malpractice. Therefore, health policymakers can reduce errors and failures by adopting continuing education on medical, ethical, and legal issues.

Keywords: Medical malpractice, Iran, Health system

Conflicts of Interest: None declared

Funding: None

*This work has been published under CC BY-NC-SA 1.0 license.

Copyright $\odot$ Iran University of Medical Sciences

Cite this article as: Raeissi P, Taheri mirghaed M, Sepehrian R, Afshari M, Rajabi MR. Medical malpractice in Iran: A systematic review. Med J Islam Repub Iran. 2019 (14 Oct);33:110. https://doi.org/10.47176/mjiri.33.110

\section{Introduction}

Health system is one of the most important sectors of each country's socioeconomic activities, which spends a large amount of resources on meeting the health care needs annually (1). The main concern of policymakers in the health system is spending a substantial amount of money on health care, and thus they pay attention to the waste of

\footnotetext{
Corresponding author: Masood Taheri mirghaed, taherimirghaed.m@iums.ac.ir

1. Department of Health Services Administration, School of Health Services Management and Medical Information Sciences, Iran University of Medical Sciences, Tehran, Iran

2. Department of Nephrology and Cardiology, Faculty of Medicine, Shahed University, Tehran, Iran
}

resources in the health system. A part of this waste of resources is due to infringements, errors, and failures related to the provision of health services, which in general are called medical malpractice (2-4). Medical malpractice occurs when a patient comes to a physician and he/she takes the responsibility of the care and treatment, but the physi-

\section{$\uparrow$ What is "already known" in this topic:}

Medical malpractice occurs when a hospital, doctor, or other health care professionals, through a negligent act or omission, cause an injury to a patient. Previous studies have shown the rate of medical malpractice in various medical specialties separatel.

\section{$\rightarrow$ What this article adds:}

Here, the rate of medical malpractice was systematically reviewed in various specialities. Also, the reasons for this failure were investigated. 
cian performs or acts contrary to acceptable medical standards and imposes psychological, physical, and financial burden on the patient (5).

Patient care is considered an essential component of health care services and a wide range of medical and paramedical staff are involved in this field (6). Physicians have always been recognized as the first provider of care to the patient. One of the most important occupational tensions that any physician can face in his/her profession is the patients' complaint. This causes anxiety, tension, depression, isolation, and even illness in the physicians (7). Medical malpractice represents a serious problem in the health system and is one of the risk factors for patient safety (8), which damages the patient and increases costs for the patient and the health care provider (9).

On the other hand, studies have shown that increasing the number of complaints in some medical specialties reduces the willingness of candidates to enter these disciplines (10). Due to the complexity of the health system and the fact that there is no system without any fault, the incidence of medical error is inevitable in all countries (11). According to various studies, the rate of patient complaints in the world has increased in recent years (12-14). A study in 2000 showed that medical failure occurs in $5 \%$ to $18 \%$ of all hospital admissions and accounts for 98000 deaths annually (15). Another study in 2013 found that medical errors are the third most common cause of death (440 000 deaths a year) in the United States (16).

Iran is no exception in this regard and complaints from physicians are rising in the country (13). The number of complaints of medical malpractice to the medical council of Tehran province increased from 134 in 1995 to 1270 in 2005 (17). People's awareness of their individual rights (18), the increase in the complexity of the medical technology, the remarkable increase in the number of population (19), the collaboration of various groups of treatment in providing service to patients, the fatigue caused by daily workload, and the need to have a great deal of information about health systems are among the factors that increase complaints (20-22). The occurrence of medical errors and subsequent complaints and seeking compensation by patients confront the community, physicians, and the medical community with a growing crisis, and thus the destructive effects of this process are seen in the services provided by this expert group. These litigations are important to the physicians, as they can continue for a long time and cause serious problems, preventing the physicians to attend to their professional and personal life for weeks. Moreover, these processes cause financial loss, anxiety, psychological pressure, and damage the reputation of physicians, and harm the patients' trust in physicians (23).

This study was conducted to investigate the complaints against medical malpractice cases referred to Iran Medical Council in various medical fields to provide solutions to reduce the complaints and improve service delivery.

\section{Methods}

This review was performed according to the "Preferred Reporting Items for Systematic Reviews and Meta-Anal- yses" (PRISMA) guidelines (24). Two authors independently searched Embase, PubMed/MEDLINE, ISI/Web of Science (WOS), Scopus, and Iranian databases, such as MagIran, SID, and Irandoc, from 01/01/1990 up to 07/01/2018. Also, the grey literature (via Google Scholar) was searched. Studies written in English or in Persian were searched. The search strategy was as follows: (Malpractice* OR Negligence* OR medical malpractice OR physician impairment OR professional impairment) AND (Physician*OR doctor OR medical doctor OR medical practitioner OR physician associate OR physicians OR practitioner OR private physician) AND (Iran*). Medical subject headings (Mesh) and wild-card options were used where appropriate. This search strategy was planned by the help of an information specialist. In addition, reference lists of each identified study were investigated for potentially eligible studies.

\section{Study selection and data extraction}

Studies were included if they met the following criteria:

1. Study type: Studies presenting original data on malpractice claims.

2. Study setting: All of the early studies that reported the rate of medical malpractice in various medical specialties were included in the study. Studies in other medical fields, such as dentistry, nursing, and midwifery, were excluded.

3. Outcome: Claim against medical malpractice, defined as a written demand for compensation because of medical injury.

4. Published in English or Persian language.

\section{Data extraction}

Two authors independently extracted the following information from selected studies: (1) prevalence of claim against medical malpractice; (2) type of physician's specialty; (3) year of study; (4) location of the study; (4) prevalence of proven malpractice; (5) type of malpractice; and (6) study setting. Any disagreement in this regard was resolved through consensus, if not however, the corresponding author was consulted. Information was entered into the forms which had been designed for this purpose.

\section{Data synthesis}

A narrative synthesis of the included studies was planned. A narrative review is discursive in nature and seeks to summarize the current state of knowledge in relation to a particular domain by considering a wide variety of sources and reaching conclusions through reasoning or argument.

\section{Results}

Flowchart of screened, excluded, and included studies are presented in Figure 1. In this study, 28 studies on complaints against medical malpractice that were referred to the Legal Medicine Organization and Medical Council from 1990 to 2018 were considered $(12,14,18,25-44)$. A total of 3997 cases were investigated, of which $71.4 \%$ were about specialists' medical malpractice in Iran after 2009 (Fig. 2).

Most studies about medical malpractice were conducted 


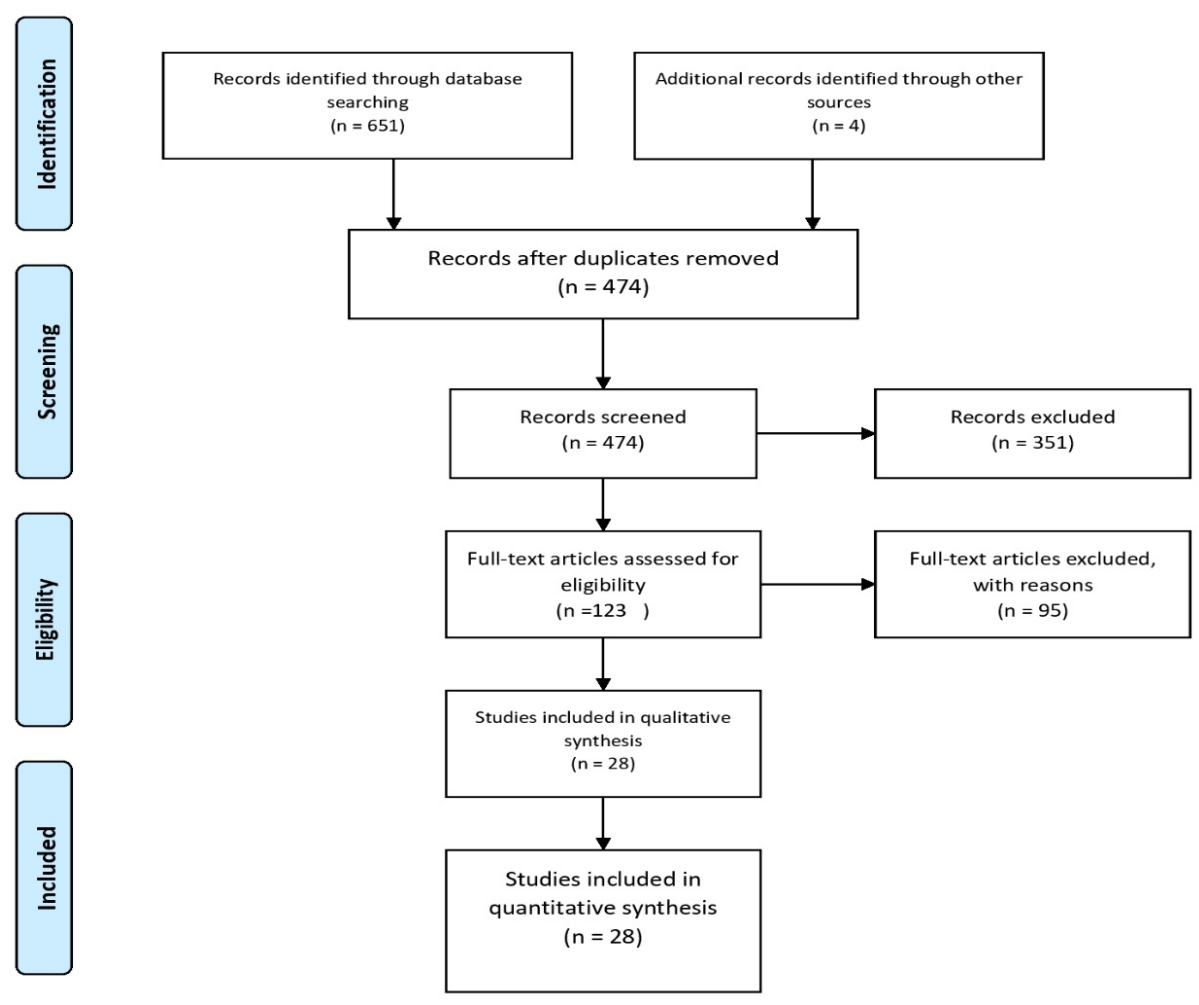

Fig. 1. Flowchart of screened, excluded, and included studies

in the provinces of Tehran, Kermanshah, and Khuzestan (Fig. 3).

Nearly 1455 cases $(36 \%)$ were proved to be medical malpractice, and physicians were acquitted in 2542 (64\%) cases. Most complaints were from gynaecologists, accounting for $43 \%$ of all complaints, followed by orthopaedic specialists who ranked second, with $21.4 \%$ of the total complaints. General surgeons and ophthalmologists were also subject to the highest number of complaints in the next ranks. The lowest number of complaints belonged to psy- chiatrists and paediatricians, with $0.6 \%$ and $0.9 \%$, respectively (Table 1).

The most type of failure was due to lack of skill (30.4\%), followed by negligence (29.2\%). Imprudence (26.3\%) and noncompliance with governmental requirements $(14.1 \%)$ were also in the next ranks, respectively (Table 2). The highest number of failures occurred in private hospitals, accounting for $34.2 \%$ of the total proved failures. Subsequently, $22.4 \%$ of failures occurred in nonteaching public hospitals, and $21.5 \%$ of the total proved failures were in governmental teaching hospitals. Also, $12.9 \%$ of failures

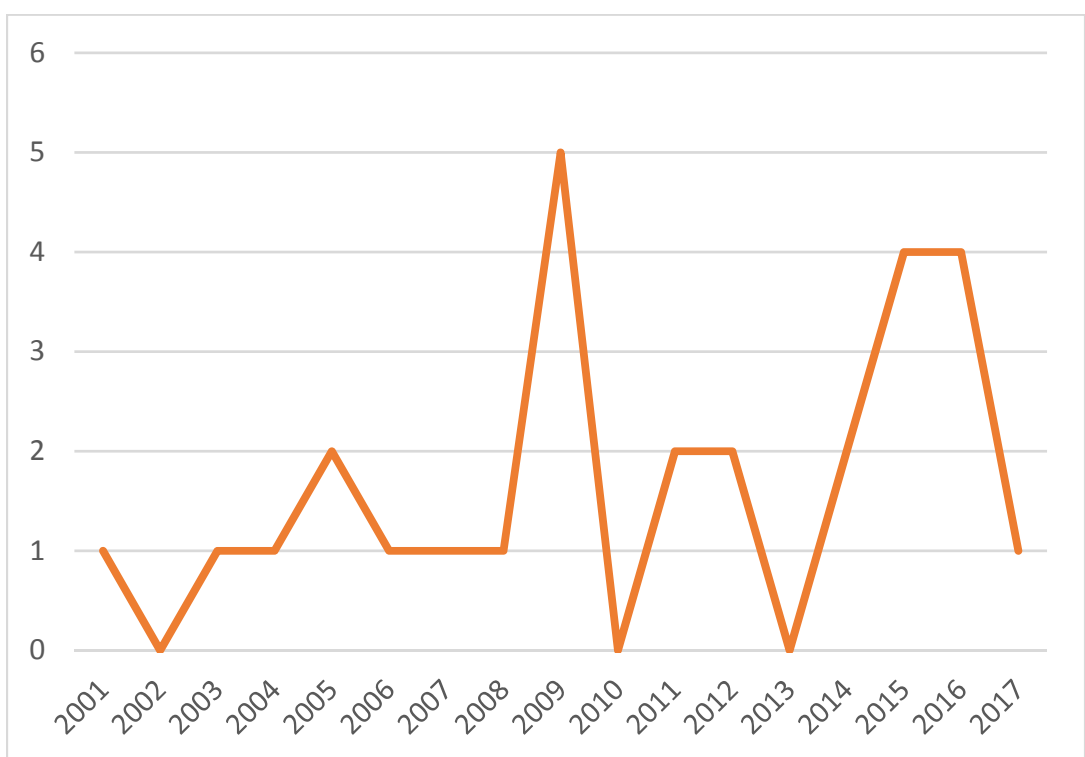

Fig. 2. Frequency distribution of medical malpractice studies in Iran by year of publication 


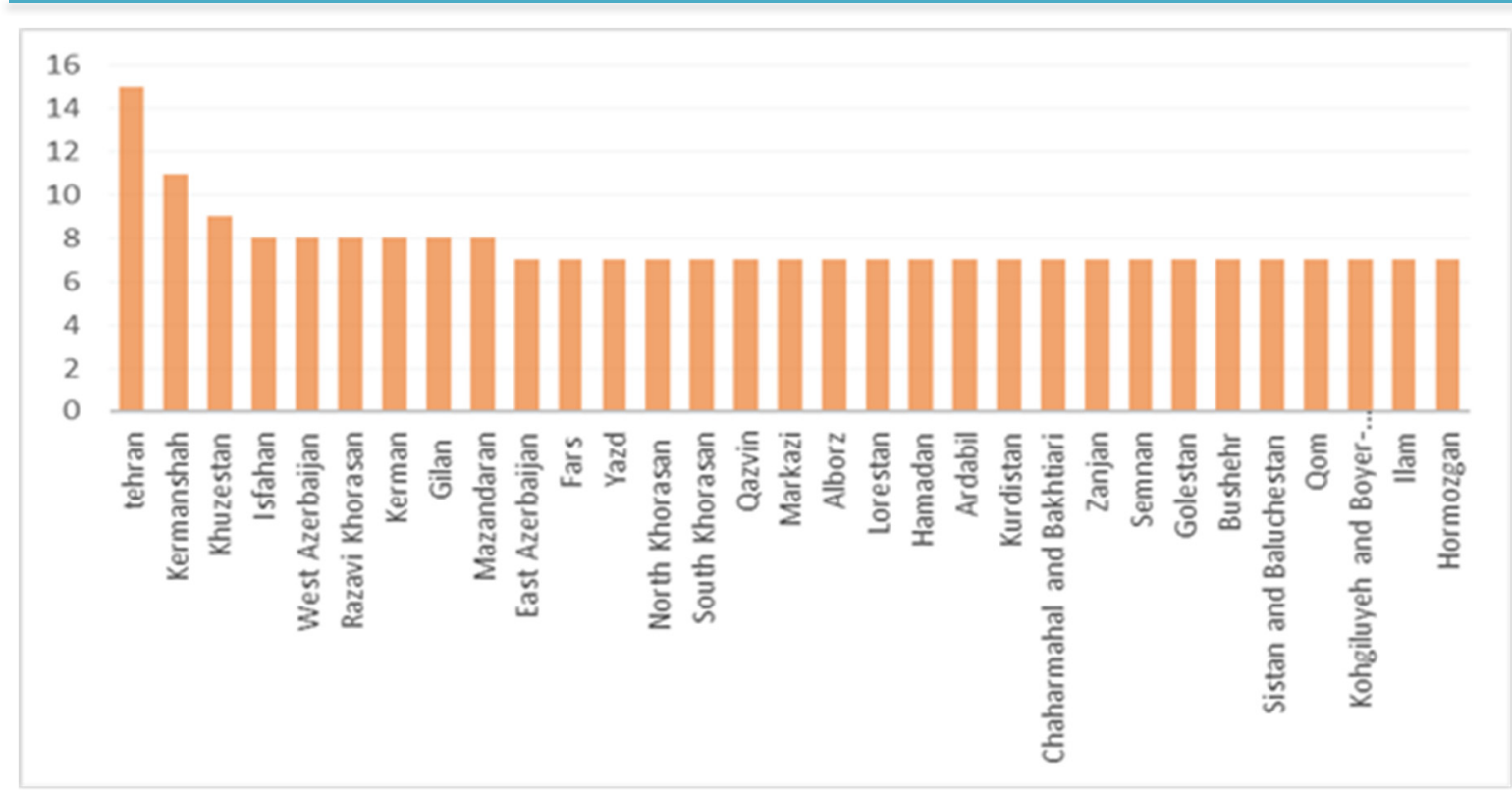

Fig. 3. Frequency of medical malpractice publications based on the provinces of the country

Table 1. Distribution of medical malpractice cases by specialty in Iran $(\mathrm{n}=3997)$

\begin{tabular}{|c|c|c|c|c|c|c|}
\hline \multirow[b]{2}{*}{ Area of specialty } & \multicolumn{2}{|c|}{ Liable } & \multicolumn{2}{|c|}{ Not liable } & \multicolumn{2}{|c|}{ Total } \\
\hline & $\mathrm{N}$ & $\begin{array}{c}\% \text { of total } \\
\text { specialty }\end{array}$ & $\mathrm{N}$ & $\begin{array}{c}\% \text { of total } \\
\text { specialty }\end{array}$ & $\mathrm{N}$ & $\begin{array}{c}\% \text { of total } \\
\text { cases }\end{array}$ \\
\hline Orthopaedics & 334 & 39 & 518 & 61 & 852 & 21.4 \\
\hline Skin and cosmetics & 73 & 55 & 60 & 45 & 133 & 3.4 \\
\hline Emergency Medicine Specialists & 53 & 65 & 29 & 35 & 82 & 2 \\
\hline Anaesthesiology & 140 & 60 & 92 & 40 & 232 & 5.9 \\
\hline General surgeons & 157 & 44 & 196 & 56 & 353 & 8.8 \\
\hline Ophthalmologists & 73 & 21 & 274 & 79 & 347 & 8.6 \\
\hline General physicians & 60 & 55 & 50 & 45 & 110 & 2.7 \\
\hline Gynaecologists & 497 & 29 & 1221 & 71 & 1718 & 43 \\
\hline Psychiatrics & 17 & 71 & 7 & 29 & 24 & $0 / 6$ \\
\hline Paediatrics & 8 & 22 & 29 & 78 & 37 & $0 / 9$ \\
\hline Cardiovascular surgery & 27 & 48 & 29 & 52 & 56 & 1.4 \\
\hline Neonatology & 16 & 30 & 37 & 70 & 53 & 1.3 \\
\hline Total & 1455 & 36 & 2542 & 64 & 3997 & 100 \\
\hline
\end{tabular}

Table 2. Distribution of type of proven medical malpractice

\begin{tabular}{lc}
\hline Type of malpractice & \% of total malpractice \\
\hline Negligence & 29.2 \\
Lack of skills & 30.4 \\
Lack of obedience to governmental regulations & 14.1 \\
Imprudence & 26.3 \\
Total & 100 \\
\hline & $\%$ of total malpractice \\
Table 3. Distribution of proven medical malpractice by setting & 12.9 \\
\hline Setting & 22.4 \\
\hline Private clinic & 34.2 \\
Government hospital & 2.2 \\
Private hospital & 21.5 \\
Government clinic & 2.1 \\
University hospital & 4.7 \\
Charity & 100 \\
Other & \\
Total & \\
\hline
\end{tabular}

occurred in private clinics, $2.1 \%$ in charity centers, and $4.7 \%$ in other health centers (Table 3 ).

\section{Discussion}

The Medical Council in Iran is responsible for investigating the professional misconduct of physicians and other relevant professions. Medical council examines the profes- sional misconduct of physicians through a regulation entitled "disciplinary regulation for investigating the guild and professional misconduct of medical and associate professions". This regulation is the most significant legal document available on examples of medical misconduct in the country. The aim of this study was to investigate the failure of medical specialists in Iran over the past 3 decades. A total of 28 studies have investigated the failures of medical 
specialists in Iran during this period, and 3997 complaint cases of medical malpractice have been referred to the Legal Medical Organization and Medical Council in these studies.

The findings of this study showed that $36 \%$ of complaints of medical malpractice were confirmed by the Medical Council. In a study conducted at a paediatric health research center in the United States during 1985 and 2005, it was found that $25 \%$ of complaints from urologists were medical malpractice (45). In European countries, 1 in every 6 physicians is prosecuted for medical misconduct. This rate is constantly increasing in Europe, and thus defensive medicine is becoming a global phenomenon in Europe (46). Complaints from the physicians have psychological consequences, such as stress and anxiety (47), occupational consequences, such as resort to the jurisdictions and absenteeism $(48,49)$, and social consequences, such as the impact on the reputation (50) of the physicians. The change in the practice of medicine toward defensive medicine (51), conservatism, and doctors' fear of lawsuits, which may negatively affect the medical care, are other consequences. Defensive medicine also causes induced demand and imposes additional costs on patients $(52,53)$. Therefore, it is advisable to investigate complaints in preliminary commissions before referring to jurisdictions, and if necessary, summon the physician (54).

Gynaecologists were the most specialists subject to complaints (43\%), followed by orthopaedic specialists $(21.4 \%)$. The results of this study are consistent with the findings of studies conducted in Turkey (54), Taiwan (55), Germany (56), United Kingdom (57), USA (58), and Saudi Arabia (59), in which gynaecologists had the highest number of complaints. The surgical team is always the main target of medical complaints due to the invasive nature of their medical practices (60). In a study in Spain, most complaints have been reported against gynaecologists, orthopaedist, neurosurgeons, ophthalmologists, and anaesthesiologists (61). Kane in a study conducted in 2011, reported that $42 \%$ of American physicians during their work period, and $90 \%$ of surgeons 55 years and older were sued due to medical malpractice. However, the general surgeons and gynaecologists experienced twice the mentioned amount (62). The findings were also consistent with research findings in the United States (58), suggesting that the lowest number of specialists being sued are paediatricians. Medical malpractice in the field of gynaecology will lead to many problems in the future which needs to be addressed. The availability of an efficient, up to date, and moralistic system for investigating professional infringement in the provision of reproductive health services that includes a wide range of clients is a key guide to identify the violation, make a fair decision, and respect the rights of the recipient and the provider of services (63). Continuous education and training of perinatal nurses, identifying high-risk patients and their precise nursing care, improving the quality and standard of delivery through holding complaints assessment committees and precise RCAs of these types of complaints, providing health education to pregnant women, and precise completion of clinical records are among other methods of reduc- ing medical defects in the fields of gynaecology and obstetrics $(14,64,65)$.

Findings of this study showed that lack of skill (30.4\%) and negligence $(29.2 \%)$ were responsible for more than half of medical malpractices. These findings are consistent with the results of the Gundogmus (54) study, which showed that diagnostic and therapeutic errors were the most prominent factors of proved failures in Turkey. Since the lack of skill and negligence are the main causes of many medical errors, it seems that conducting retraining courses and collaborating more with scientific-research associations can somewhat reduce these errors. The lack of awareness of the medical practitioners about the legal issues and regulations governing medical practice and the responsibility of doctors towards patients is one of the main reasons for increasing the number of complaints (66). In this regard, holding workshops to teach the appropriate methods of physicians' communication with the patients and relatives, increasing the medical staff's information on the legal issues of the treatment, and emphasizing on informing the patients and their relatives about the possible and even unpredictable complications of diagnosis and treatment processes can help reduce medical complaints (67).

Negligence and lack of obedience of governmental regulations were the other reasons for the medical defects identified in this study. Providing such measures as better organization and supervision of treatment, the intensification of penalties, and more respect for medical ethics can be effective in this regard. In the study of Asad et al, the most common causes of complaint of medical malpractice by patients were delay in the diagnosis and lack of follow-up and postoperative management (68). Nicole et al, in their study, showed that $88 \%$ of the errors in orthopaedic surgeries were due to procedural errors and negligence (69). Acting based on medical guidelines and protocols, holding a counselling session to answer the questions of patients before the surgery, eliminating the financial relationship between the physician and patient completely are measures that can help prevent patient complaints and medical malpractice (7072).

The findings of this study showed that $43.9 \%$ of proved failures occurred in the public sector, including university and nonuniversity hospitals, and $37.1 \%$ of proved failures have occurred in the private sector. Referring a large percentage of people to the public sector, older diagnostictherapeutic equipment in public centers, the use of medical students at these centers, and being university public sectors that increase the likelihood of error, and giving more importance to the quality of treatment in the private sector were among the reasons for this finding.

Thus, it is essential to establish a central monitoring system that links the 3 legal medicine organizations, the medical council, and the hospital to prevent any medical error. Moreover, providing necessary training to familiarize the physicians with rules and medical errors should be provided continuously in-hospital training and retraining programs (73). The establishment of a reporting system by physicians and the central system to control medical errors will be effective in reducing medical misconduct. Moreo- 
ver, disclosure of errors is one of the most important strategies to prevent medical errors (74).

The presentation of a complaint does not always have a negative effect, as it can be used as a way to improve work processes and a solution to increase patient safety through improving the accuracy of the treatment team and increasing clinical skills (67). As long as the root causes of the error are not identified and resolved, the error will be repeated by a particular person or any other person who replaces him. Developing a systemic attitude to errors, creating the culture of organizing, and active participation of staff in meetings that examine medical errors, creating a team for this purpose, and identifying all the causes that lead to medical errors have a significant impact on the prevention of these errors.

\section{Conclusion}

The findings of this study on medical malpractice, specialists at risk, various locations of malpractice occurrence, and the types of medical malpractice can be used as a basis for redesigning new approaches to medicine and encouraging appropriate retraining programs. Improving patient and physician relationships, observing medical ethics, increasing the scientific and technical skills of the medical staff, and following the medical guidelines and protocols will prevent medical malpractice. Therefore, health policymakers can reduce errors and defects by adopting the approach of continuous education on medical, ethical, and legal issues.

\section{Ethical considerations}

Ethical issues (including plagiarism, informed consent, misconduct, data fabrication and/or falsification, double publication and/or submission, and redundancy) were thoroughly observed by the authors.

\section{Acknowledgments}

No financial support was received for this study.

\section{Conflict of Interests}

The authors declare that they have no competing interests.

\section{References}

1. Mahmoodabadi HB, Setareh M, Nejadnick M, Niknamian M, Ayoobian A. The frequency and reasons of medical errors in cases referred to Isfahan Legal Medicine Center. Health Info Manag. 2012;9(1):101-9.

2. Leape LL, Brennan TA, Laird N, Lawthers AG, Localio AR, Barnes $\mathrm{BA}$, et al. The nature of adverse events in hospitalized patients: results of the Harvard Medical Practice Study II. N Engl J Med. 1991;324(6):377-84.

3. Thomas EJ, Studdert DM, Burstin HR, Orav EJ, Zeena T, Williams EJ, et al. Incidence and types of adverse events and negligent care in Utah and Colorado. Med Care. 2000;38(3):261-71.

4. Mello MM, Brennan TA. Deterrence of medical errors: theory and evidence for malpractice reform. Tex L Rev. 2001; 80:1595.

5. Shojapoorian S, Katoozian N. Social Responsibility consequences of physicians' medical fault. Tehran: Ferdousi Publication; 1995.

6. Tafreshi PM, Abedzadeh Z. Indicator for the development of nursing care quality in Iranian. Nurse Rev. 2008; 55:399-406.

7. Jafarian A, Parsapoor A, Hajtarkhani A, Asghari F, ImamiRazavi SH, Yalda A. A survey on the complaints registered in Medical Council Organization of Tehran. J Med Ethics History Med. 2009;2(2):67-74.
8. Lebanon N, Hanover N. How many deaths are due to medical error? Getting the number right. Eff Clin Pract. 2000; 6:277-83.

9. Mahfouzi A, Zamani R. The evaluation of the causes of anesthesiologist's legal pursuits in Tehran Medical Consil from 1993 to 2003. Iran J Forensic Med. 2007;13(2):98-101.

10. Yawn B, Wellever A, Hartley D, Casey M, Krein S, Moscovice I. Availability of rural Minnesota obstetric services: is it a problem? J Rural Health. 1995;11(3):192-203.

11. Haghshenas M, Vahidshahi K, Amiri A, Rezaee M, Rahmani N, Pourhossen M, et al. Study the frequency of malpractice lawsuits referred to forensic medicine department and medical council, Sari, 20062011. J Mazandaran Univ Med Sci. 2012;21(86):253-260

12. Fallahi M, Vaghefi S, Makhmalbaf M, Afjeh S, Mostafazadeh B. Analysis of Negligence in field of Neonatology in Medical Commissions Office in Tehran, Iran. Int J Med Tox Forensic Med. 2016;6(3 (Summer)):135-41.

13. Siabani S, Alipour AA, Siabani H, Rezaei M, Daniali S. A survey of complaints against physicians reviewed at Kermanshah. J Kermanshah Uni Med Sci. 2009;13(1).

14. Akhlaghi M, Tofighi-Zavvare H, Samadi F. Complaints of malpractice in obstetrics and gynecology referred to medical commission of Legal Medicine Organization during 1380-1381 and the causes and prevention methods. Iran J Forensic Med. 2004;34:4-70.

15. Institute of Medicine. To err is human: Building a safer health system National Academy Press, Washington, DC 2000.

16. James JT. A new, evidence-based estimate of patient harms associated with hospital care. J Patient Saf. 2013;9(3):122-8.

17. Tofighi-Zavvare H, GhadiPash M. survey on medical malpractice result in death registered in the Coroners offoce of forensic medicine, province of tehran, during 1995 to 1999. Iran J Forensic Med. 2002;8(27):5-8.

18. Khara A. Descriptive Analysis of Medical Malpractice Complaints from General Surgeons in Tehran 2012 - 2013; a Short Report. J Police Med. 2016;5(1):1-6.

19. Sheikh Azadi A, Ghadyani M, Kiani M. The Investigation Methods to Dentistry Malpractices in Iran. Ir J Forensic Med. 2007;13(3):17180 .

20. Shojania KG, Wald H, Gross R. Understanding medical error and improving patient safety in the inpatient setting. Med Clin North Am. 2002;86(4):847-67.

21. Whipple JK, Quebbeman EJ, Lewis KS, Gaughan LM, Gallup EL, Ausman RK. Identification of patient-controlled analgesia overdoses in hospitalized patients: a computerized method of monitoring adverse events. Ann Pharmacotherapy. 1994;28(5):655-8.

22. Blendon RJ, DesRoches CM, Brodie M, Benson JM, Rosen AB, Schneider E, et al. Views of practicing physicians and the public on medical errors. N Engl J Med .2002;347(24):1933-40.

23. Leaman TL, Saxton JW. Preventing Malpractice: The Co-active Solution: Springer Science \& Business Media; 2013.

24. Liberati A, Altman DG, Tetzlaff J, Mulrow C, Gøtzsche PC, Ioannidis JP, et al. The PRISMA statement for reporting systematic reviews and meta-analyses of studies that evaluate health care interventions: explanation and elaboration. PLoS Med. 2009;6(7):e1000100.

25. BarzgarBafrouee A, Zarenezhad M, Gholamzadeh S, Hosseini SMV, Gholi A, Gharedaghi J, et al. Evaluation of Orthopedic Specialists' Malpractice Complaints Referred to Legal Medicine Organization from 2008 to 2010. Iran J Forensic Med. 2016;22(1):55-66.

26. Pourali M, Shojaee M, Amini A, Alimohammadi H, Hatamabadi H. 82 Cases of Medical Lawsuit against Emergency Medicine Specialists\&59; a case study. Ir J Emerg Med. 2015;2(3):122-6.

27. Bastani M, Hejazi A, Zarenejad M, Shahriary S, Sahmeddini MA. Malpractice cases of anesthesiology leading to death or impairment referring to Shiraz forensic medicine commissions during 2006-2011. Razi J Med Sci. 2014;21(122):61-7.

28. Sadr SS, Ghadyani MH, BagherZadeh AA. Assessment of records of complaints from medical malpractice in the field of orthopedic, in the coroner's Office of Forensic Medicine, province of Tehran, during 1988 to 2003. Ir J Forensic Med. 2007;13(2):78-86.

29. Nilforoushzadeh MA, Rahmanian M, Mardani F, Heidari A. The causes of complaints related to skin and cosmetics in general offices of legal medicine in Isfahan Province, Iran, during 2002-2013. J Isfahan Med School. 2015;2015:8.

30. Haghghi Z, Esmaeeli H, Attaran H. Analysis of 100 malpractice claims against surgical team judged at Mashad medical council. Iran J Surg. 2005;13(3-4):19-30. 
31. Parhizgar SH, Fayyaz AF. Investigation of complaints of medical malpractice from ophthalmologists referring to the country's legal medicine organization between 2000 and 2001. Iran J Forensic Med. 2005;11(2):70-68.

32. Moin A, Ostad Ali Makhmalbaf M, Davati A. Study of medical malpractice complaints in the field of dermatology and cosmetic, in the coroner's office of Forensic Medicine, province of Tehran, during 2002 to 2010. J Dermatol Cos. 2011;2(1):17-29.

33. Rafizadeh Tabai Zavareh SM, Haj Manoochehri R, NasajiZavareh M. Frequency of failure of general practitioners in referral complaints to the Tehran Forensic Medicine Commission from 2003 to year. Iran J Forensic Med. 2007;13(3):152-7.

34. Mahfoozi A, TaghdisiNezhad F, Abedi H. Investigating cases of medical malpractice in anesthesia referral to the Commission of the Legal Medicine Organization of the country at the age of six years ending March 1999. Iran J Forensic Med. 1999;26(8):4-10.

35. Kamiab F, Afshari P, Keikhahi B, Tadayon M, VahabiShekarloo T, Haghighizadeh MH. A Review of Reasons of Malpractice of Obstetricians, Gynecologists and Midwives. Iran J Forensic Med. 2016;22(3):165-71.

36. Mansoori M, Tabatabaie A, Samimi M. Evaluation of Malpractice Claims against Ophthalmologists Referred to the Iran Medical Council during Years 2003 to 2008. Bina J Ophthamol. 2012;17(4):374-9.

37. Mahfouzi A, Zamani R. The evaluation of the causes of anesthesiologist's legal pursuits in Tehran Medical Consil from 1993 to 2003. Iran J Forensic Med. 2007;13(2):98-101.

38. Azimi K, Poorbakhtiar M, Taghizadeh Z, Soltani K, Ghadipasha M, Daneshparvar H. A Survey on Various Types of Medical Malpractice in Relation with Pregnancy and Delivery, Referred to Relevant Commissions of the Iranian Legal Medicine Organization. Iran J Forensic Med. 2017;23(2):132-41.

39. Sadr SS, Ghadyani MH, Avish S, Ramim T. Medical malpractice frequency in the psychiatric field, in Medical Coun-cil of Iran, from 2001 to 2010: a brief report. Tehran Uni Med J. 2014;72(5):329-34.

40. Hejazi S, Zeynali M, Farokh H. study of pediatric malpractice claims registered at urmia medical council during 10-year period (1375-1385). Urmia Med J. 2009;20(2):123-30.

41. Siabani S, Alipour AA, Siabani H, Rezaei M, Daniali S. A survey of complaints against physicians reviewed at Kermanshah Medical Council 2001-2005. J Kermanshah Uni Med Sci. 2009;13(1):e79831.

42. Badakhsh H. Malpractice claims of Gynecologists received by Medical Council, Tehran, 1992-96. Feyz J Kashan Uni Med Sci. 2003;6(4):76-81.

43. Kazemi S, Mostafazadeh B, Heshmati S, Emamhadi M. Medical Malpractice in Cardiovascular Surgery; Cases Referred to Tehran Forensic Medicine Organization during 2011-13. Int J Med Tox Forensic Med. 2015;5(4 (Autumn)):207-13.

44. Daneshparvar H, Javadian A. Malpractice Complaints against Ophthalmologists Referred to the State of Legal Medicine Organization in Iran. Iran J Ophthalmol. 2008;20(1):4-8.

45. Perrotti M, Badger W, Prader S, Moran ME. Medical malpractice in urology, 1985 to 2004: 469 consecutive cases closed with indemnity payment. J Urol. 2006;176(5):2154-7.

46. Inelmen E. On clinical errors in geriatric medicine diagnosis: ethical issues and policy Implication. Ethics Med. 2010;26(1):15-24.

47. Bourne T, Wynants L, Peters M, Audenhove CV, Timmerman D, Calster BV. The impact of complaints procedures on the welfare, health and clinical practice of 7926 doctors in the UK: a cross-sectional survey. BMJ Open. 2015 Jan 15;5(1):e006687.

48. Allosop J, Mulcahy L. Maintaining professional identity: doctors' responses to complaint. Soc Health Ill. 1998; 20 (6):802-824.

49. Hubbard F. The Physicians' Point of View Concerning Medical Malpractice: A Sociological perspective on the Symbolic Importance of Tort Reform. Geo Law Rev. 1989; 23(2): 295

50. Ciofu C. The frequency and severity of medical malpractice claims: high risk and low risk specialties. Maedica (Buchar). 2011;6(3):230 231 .

51. Hershey N. The Defensive Practice of Medicine. Law and Ethics in Health Care. Cambridge: MIT Press 1982.

52. Thomas J. The Effect of Medical Malpractice. Ann Health Law Adv Direct. 2010;19:306-315.

53. Kazemi S, Riahi ME. Individual and Social Impacts of Medical Malpractice Complaints on doctors: A qualitative study. Com Health. 2018;5(2):159-70.
54. Gundogmus UN, Erdogan MS, Sehiralti M, Kurtas O. A descriptive study of medical malpractice cases in Turkey. Ann Saudi Med. 2005;25(5):404-8.

55. Hwang CY, Wu CH, Cheng FC, Yen YL, Wu KH. A 12-year analysis of closed medical malpractice claims of the Taiwan civil court: A retrospective study. Medicine. 2018;97(13): e0237.

56. Knaak JP, Parzeller M. Court decisions on medical malpractice. Int J Leg Med. 2014;128(6):1049-57.

57. Jena AB, Seabury S, Lakdawalla D, Chandra A. Malpractice risk according to physician specialty. New Eng J Med. 2011;365(7):629-36.

58. Carroll AE, Buddenbaum JL. High and low-risk specialties experience with the U.S. medical malpractice system. BMC Health Serv Res. 2013;13:465.

59. Alsaddique AA. Medical liability. The dilemma of litigations. Saudi Med J. 2004;25(7):901-6.

60. Deshpande SP, Deshpande SS. Factors impacting perceived threat of malpractice lawsuits by various medical specialists. Health Care Manag. 2011;30(1):55-65.

61. Trilla A, Bruguera M. Spain: avoiding lawsuits. The Lancet. 2000;356(9237):1266-7.

62. Kane C. Policy research perspectives: medical liability claim frequency: a 2007-2008 snapshot of physicians. Am Med Assoc. 2010.

63. Farajkhoda T, Latifnejad Roudsari R, Abbasi M. Assessment of an effective observer system in SRH malpractice. Med Ethics J. 2011;5(16): 133-162.

64. Quam L, Dingwall R, Fenn P. Medical malpractice claims in obstetrics and gynaecology: comparisons between the United States and Britain. Br J Obstet Gynaecol. 1988 May;95(5):454-61.

65. Glaser LM, Alvi FA, Milad MP. Trends in malpractice claims for obstetric and gynecologic procedures, 2005 through 2014. Am J Obstet Gynecol. 2017;217(3):340. e1-340.e6.

66. Rafiezade Tabatabei Zavare SM, Haj Manuchehrei R, Nasaji Zavare M. Survey on General Practice Errors in Referred Sues to the Commission of Tehran Legal Medicine Center from 2003-2004. Iran J Forensic Med. 2007;13(3):152-157.

67. Aghakhani N, Cheraghi R, Zarei A, Alinejad V, Nazimi N, Rahbar N, et al. Study of Causes of Health Providers' Malpractices in Records Referred to Forensic Medicine Organization in Urmia, during 20092013. Med Law J. 2017;11(42):83-100.

68. Choudhry AJ, Haddad NN, Martin M, Thiels CA, Habermann EB, Zielinski MD. Medical Malpractice in Bariatric Surgery: a Review of 140 Medicolegal Claims. J Gastrointest Surg. 2017;21(1):146-54.

69. Rynecki ND, Coban D, Gantz O, Gupta R, Ayyaswami V, Prabhu AV, et al. Medical Malpractice in Orthopedic Surgery: A WestlawBased Demographic Analysis. Orthopedics. 2018.

70. Parks-Savage A, Archer L, Newton H, Wheeler E, Huband SR. Prevention of medical errors and malpractice: Is creating resilience in physician's part of the answer? Int J Law Psychiatry. 2018 Sep 1;60:35-9.

71. Razavi SM, Khajehnasiri F, Dabiran S. Prevention of Malpractice in the Field of Health, as the Other Type of Prevention. J Prev Med. 2017 Sep 28;7(09): 183 .

72. Birkeland S, Bogh SB. Education trajectories and malpractice complaints-A study among Danish general practitioners. Cogent Edu. 2018 Jan 1;5(1):1-6.

73. Safdari R, Masuri N. The importance of electronic proprietary registration system for referral complaints due to medical malpractice in the country's forensic medicine organization. Iran J Forensic Med. 2005;11(2):99-106.

74. Matlow A, Stevens P, Harrison C, Laxer RM. Disclosure of medical errors. Pediatr Clin North Am. 2006;53(6):1091-104. 